\title{
Identification, Characterization and Expression of Methuselah-Like Genes in Dastarcus helophoroides (Coleoptera: Bothrideridae)
}

\author{
Zhengqing Zhang ${ }^{1,+}{ }^{+}$Huapeng Wang ${ }^{1,+}$, Chunfeng Hao ${ }^{1}$, Wei Zhang ${ }^{1}$, Miaomiao Yang ${ }^{2}$, \\ Yong Chang ${ }^{1}$ and Menglou $\mathrm{Li}^{1}{ }^{1 \text { ** }}$ \\ 1 Laboratory of Forestry Pests Biological Control, College of Forestry, Northwest Agriculture and \\ Forestry University, Yangling 712100, China; zhangzhengqing0802@hotmail.com (Z.Z.); \\ hppwang@163.com (H.W.); hlzsdau2009@163.com (C.H.); zhangwei1062002@163.com (W.Z.); \\ changyongzzzx@163.com (Y.C.) \\ 2 Institute of Biotechnology, Shaanxi Xueqian Normal University, Xi'an 710100, China; yangmmgg@163.com \\ * Correspondence: limenglou@hotmail.com; Tel.: +86-29-87082125 \\ + These authors contributed equally to this study. \\ Academic Editor: Paolo Cinelli \\ Received: 13 March 2016; Accepted: 17 October 2016; Published: 21 October 2016
}

\begin{abstract}
Dastarcus helophoroides, which has a relatively longer lifespan compared to other insects, is one of the most effective natural enemies of many large-body long-horned beetles. Methuselah (Mth) is associated with the lifespan, stress resistance, and reproduction in Drosophila melanogaster, but Mth is not present in non-drosophiline insects. A number of methuselah-like genes (mth-likes, mthls) have been identified in non-drosophiline insects, but it is still unknown whether they are present in Dastarcus helophoroides. We identified three novel mth-like genes in D. helophoroides: $m$ th-like1, mth-like2, and $m$ th-like5, and carried out bioinformatic analysis based on the full-length nucleic acid sequences and deduced amino acid sequences. Real-time quantitative polymerase chain reaction (RT-qPCR) showed variations in expression patterns of $m$ th-like genes in different tissues (highly expressed in reproductive systems) and at different developmental stages, indicating that $m$ th-likes were likely be involved in reproduction and development. The altered mRNA expression in aging adults and under oxidation, high temperature, and starvation stress, indicated that $m$ th-like genes were likely to be involved in aging and the resistance of oxidation, high temperature, and starvation. These results characterize, for the first time, the basic properties of three $m$ th-like genes from $D$. helophoroides that probably play important roles in development, aging, reproduction, and stress resistance.
\end{abstract}

Keywords: Dastarcus helophoroides; mth-like genes; expression profile; development; lifespan; reproduction; stress resistance

\section{Introduction}

Methuselah (Mth), a G protein-coupled receptor (GPCR), was originally identified in Drosophila melanogaster. In D. melanogaster, mth mutation has been shown to extend the lifespan by $35 \%$, and increase resistance to starvation, high temperatures, and paraquat treatment [1]. GPCRs are a large and important group of receptor proteins involved in signal transduction. They are classified into five large families, namely glutamate-like receptors, rhodopsin-like receptors, adhesion, frizzled, and secretin-like receptors [2]. Mth is a subfamily of the secretin-like family (family B) that has been reported as being insect-specific [3]. Mth shows an N-terminal Mth ectodomain and a C-terminal seven transmembrane (7tm) domain [4-7]. Family B of the GPCRs is a small, but structurally and functionally diverse, group of proteins that includes receptors for polypeptide hormones, molecules thought to mediate intercellular interactions at the plasma membrane and a group of Drosophila proteins 
that regulate stress responses and longevity [6]. Like other class B GPCRs, the large (195 residue) amino-terminal extracellular domain of Mth is essential for ligand-binding [6]. However, the sequence similarity of the mth gene to other class B GPCRs is observed solely within the transmembrane regions [1]. The crystal structure shows that the Mth ectodomain consists primarily of $\beta$-sheets [5], revealing a topology distinct from that of other hormone receptors [8,9].

However, mth mutation has an offsetting negative effect on fertility in D. melanogaster, which demonstrates the trade-off effect between longevity and fertility $[10,11]$. Complete knock-out of $m$ th has been reported to result in lethality of the embryo, which illustrates the crucial function of Mth receptors to maintain normal growth and development [12-14]. It has also been reported that Mth receptors were involved in the regulation of the male germline stem cell population and the sensorimotor functions in adult Drosophila $[15,16]$. The Mth receptor also has the function of strengthening the Drosophila neural synaptic transmission efficiency. Previous study has demonstrated that mutations in the $m$ th gene reduced evoked neurotransmitter release by $\sim 50 \%$, and decreased synaptic area and the density of docked and clustered vesicles [12]. As research continues, more physiological functions of Mth receptors will gradually be found.

In addition to $m$ th, a large number of methuselah-likes ( $m$ th-likes, $m$ thls) have been suggested as being present in insects. So far, fifteen $m$ th-like genes from $D$. melanogaster, seven from Anopheles gambiae, four from Bombyx mori, four from Apis mellifera, three from Acyrthosiphon pisum, and five from Tribolium castaneum have been identified [4,17-20]. Mth and mth-likes are believed to have arisen from one ancestral gene and to have been duplicated in insects [20], but it is unknown whether they share similar functions in insects.

Dastarcus helophoroides (Faimaire) (Coleoptera: Bothrideridae) is one of the most effective natural enemies of many large-body long-horned beetles, including Anoplophora glabripennis, Monochamus alternatus, Batocera horsfieldi, and Massicus raddei [21]. D. helophoroides larvae are ecto-parasitoids of late instar larvae, pupae, and young adults of long-horned beetles, which makes it a potential biological control agent for pest management [22-24]. In addition, D. helophoroides is also a suitable insect for aging studies due to the long lifespan of adults. Under laboratory conditions, D. helophoroides can live for more than eight years with continued sexual reproduction [25], providing a unique resource for molecular and physiological studies of development and reproduction. Although the morphology and physiology of $D$. helophoroides have been widely studied, the underlying molecular mechanism of development and reproduction remain unknown [26].

Thanks to sequencing and annotation of the D. helophoroides transcriptome using Illumina HiSeq 2000 (Beijing Genomics Institute, BGI, Shenzhen, Guangdong, China) sequencing technologies, genes involved in development, aging, reproduction, and resistance to adversity can be easily identified. The aim of this study, therefore, is to identify, characterize, and determine the expression profiles of $m$ th-like genes in D. helophoroides. Our expression analyses of $m$ th-likes in different tissues, at different life stages, in adults differing in age, and in adults under the stress of oxidation, high temperature, and starvation, could provide the basis for further study about their possible functions in D. helophoroides and other insects.

\section{Materials and Methods}

\subsection{Insects}

The larvae, pupae, and adults of D. helophoroides were obtained from a laboratory colony (the laboratory of Forestry Pests Biological Control, College of Forestry, Northwest Agriculture and Forestry University, Yangling, China), and were maintained in a temperature-controlled room at $23 \pm 1{ }^{\circ} \mathrm{C}, 70 \%-80 \%$ relative humidity $(\mathrm{RH})$ with a photoperiod cycle of $16 \mathrm{hL} / 8 \mathrm{hD}[25,27]$. The larvae of $D$. helophoroides were reared in test tubes using the alternative host, the pupae of Tenebrio molitor, while adults were reared in plastic boxes and fed on an artificial diet that predominantly consisted of silkworm pupa powder, sugar, yolk, agar, and water [26]. 


\subsection{Identification and Rapid Amplification of cDNA Ends}

The mth-like genes were searched using the $D$. melanogaster mth sequence as a query in the sequences from the adult $D$. helophoroides transcriptome database (previously published under the accession GBCX00000000) that had already been identified and annotated by Blastx [28] against the non-redundant (NR) protein database at the National Center for Biotechnology Information (NCBI) with a cut-off E-value of $1.0 \times 10^{-5}$ [27,29]. Based on the cDNA fragments obtained by searching the transcriptome annotation files, gene-specific primers were designed using Primer Premier 5.0 (Premier Biosoft International, Palo Alto, CA, USA) to amplify the full length $m$ th-likes of $D$. helophoroides. The primers used for PCR-based cloning are shown in Table 1. The rapid amplification of cDNA ends PCR (RACE-PCR) technique was applied to obtain the full-length mth-like cDNAs with a SMARTer ${ }^{\mathrm{TM}}$ RACE cDNA Amplification Kit (Clontech, Beijing, China) according to the manufacturer's instructions. Total RNA isolated from adult insects using a UNIQ-10 Trizol RNA extraction kit (Sangon, Shanghai, China) was used to obtain cDNA templates needed in RACE-PCR. The PCR products were cloned into the pMD-19-T Vector (TaKaRa, Kyoto, Japan) and sequenced by Sangon Biotech (Shanghai, China).

Table 1. Gene-specific primers used for rapid amplification of cDNA ends and real-time quantitative PCR of Dastarcus helophoroides mth-like genes.

\begin{tabular}{|c|c|}
\hline Oligo Name & Oligo Sequence $\left(5^{\prime}\right.$ to $\left.3^{\prime}\right)$ \\
\hline \multicolumn{2}{|c|}{ Specific primer for $5^{\prime}$ RACE } \\
\hline Mth-like1 5'GSP1 & TCACCAATAGATGGAAGTCCCTGAGAGC \\
\hline Mth-like1 5'GSP2 & CGCCGATTGGACCGTAGAAGAATGTAAG \\
\hline Mth-like2 5'GSP1 & CAACAGTTGTGGTGTGCCATCTCTAATAAA \\
\hline Mth-like2 5'GSP2 & CAAGAAGGACAGCCATACCCCAAGCATA \\
\hline Mth-like5 5'GSP1 & TTGGAGAACGACGACAGGAAGAACAGC \\
\hline Mth-like5 5'GSP2 & ACGACCAAGCGTATGCCGAATAGTAGC \\
\hline \multicolumn{2}{|c|}{ Specific primer for $3^{\prime}$ RACE } \\
\hline Mth-like1 3'GSP1 & TCTTCTCATCGGCGACATTCTCCTCTGC \\
\hline Mth-like1 3'GSP2 & ATTCTTCTACGGTCCAATCGGCGTTCTT \\
\hline Mth-like2 $3^{\prime}$ GSP1 & AGTTGGTATCATCCGGAAGTTGGCAGAG \\
\hline Mth-like2 $3^{\prime}$ GSP2 & CTTTATTAGAGATGGCACACCACAACTG \\
\hline Mth-like5 3'GSP1 & GTGGCTGTTCTTCCTGTCGTCGTTCTC \\
\hline Mth-like5 3'GSP2 & GCTGGGGTCCGTCCAAAGGAGGTGATA \\
\hline \multicolumn{2}{|c|}{ Specific primer for qPCR } \\
\hline Mth-like1 S & GCCAACCACAAGTCCTATCAGCT \\
\hline Mth-like1 A & CACCAATAGATGGAAGTCCCTGA \\
\hline Mth-like2 S & TTTGGTTAGGATTCAGCGGACT \\
\hline Mth-like2 A & GGACAGCCATACCCCAAGCA \\
\hline Mth-like5 S & ACTATTCGGCATACGCTTGGTC \\
\hline Mth-like5 A & CAGTGATCCGATTTCTTCCCG \\
\hline \multicolumn{2}{|c|}{ Reference primer for qPCR } \\
\hline$\alpha$-tubulin S & TCGGTGGTGGTACTGGGTCT \\
\hline$\alpha$-tubulin A & ACGGCTGTTGAAACTTGAGGA \\
\hline $\mathrm{EF}-1 \alpha \mathrm{S}$ & TCCTTCAAATATGCCTGGGTACT \\
\hline $\mathrm{EF}-1 \propto \mathrm{A}$ & AAATCTCTGTGTGTCCAGGGGCAT \\
\hline
\end{tabular}

\subsection{Sequence and Phylogenetic Analysis}

Open reading frames (ORF) were identified and translated with online ORF Finder software [30]. The cDNA sequences were compared with the other similar mth-like genes registered in GenBank using Blastx (searching the protein database using a translated nucleotide query) [28]. The sequence alignment and identity analysis were carried out using the DNAMAN software 
package (Lynnon Corporation, Pointe-Claire, QC, Canada) [31]. The molecular weights and isoelectric points of predicted proteins were calculated by the SWISS-PROT (ExPASy server) program "Compute $\mathrm{pI} / \mathrm{Mw}^{\prime}$ " [32]. The evolutionary relationships within the mth-like family were inferred using the neighbor-joining (NJ) method implemented in MEGA 5 (Center for Evolutionary Medicine and Informatics, Tempe, AZ, USA) with default settings and bootstrap support based on 1000 iterations [33].

\subsection{Expression Analysis through RT-qPCR}

To investigate the expression patterns of $m$ th-like transcripts at different life stages larvae of each instar (1st-6th instar), pupae, and newly-emerged adults of both male and female were collected. To investigate tissue-specific expression of $m$ th-like transcripts in D. helophoroides, the newly-emerged adults were used and the following tissues which cover the whole insect were isolated for real-time quantitative polymerase chain reaction (RT-qPCR) analysis: head, thorax, midgut, hindgut, male reproductive system, female reproductive system, and residual body (mainly body wall and muscles). The tissues were stored in RNA storage solution (CWBIO, Beijing, China) until use in RNA isolation and cDNA synthesis. To explore the expression patterns of mth-like transcripts during aging, adults fed with artificial diets were also collected and then classified into eight groups (aged 2, 4, 10, 12, $18,20,26$, and 30 months) according to the survival time from emergence. Each group contains an equal number of adult females and males (1:1). To analyze the mRNA expression profiles of $m$ th-like genes in adult $D$. helophoroides under oxidative stress, males and females (two months old) were collected at specific times $(0,1,2,3,4,5,6,12,18$, and $24 \mathrm{~h})$ after exposure to $20 \mathrm{mM}$ paraquat. To analyze the mRNA expression profiles of $m$ th-likes in adult $D$. helophoroides under high temperature stress, males and females (two months old) were collected at specific times (0, 0.5, 1.0, 1.5, 2.0, 3.0, 6.0, and $12.0 \mathrm{~h})$ after exposure to the high temperature of $45^{\circ} \mathrm{C}$. To analyze the mRNA expression profiles of $m$ th-like genes in adult $D$. helophoroides under starvation stress, males and females (two months old) were collected at specific times $(0,2,4,6,8,10,12$, and 14 days) after exposure to the condition without food, but with water. All samples that were collected were frozen in liquid nitrogen and stored at $-80^{\circ} \mathrm{C}$.

Total RNA of each sample above was isolated with a UNIQ-10 Column Trizol Total RNA Isolation Kit (Sangon Biotech, Shanghai, China) according to the manufacturer's protocol. The concentration and quality of total RNA were determined by spectrophotometry using a Maestro-NANO UV spectrophotometer (MaestroGen, Las Vegas, NV, USA). The first-strand cDNA was synthesized with a PrimeScript RTreagent Kit with gDNA Eraser (TaKaRa Bio Inc., Dalian, China) according to the manufacturer's instructions using $1 \mu \mathrm{g}$ total RNA in a $20 \mu \mathrm{L}$ final reaction volume. The cDNA was stored at $-20^{\circ} \mathrm{C}$. In accordance with reference gene selection in $D$. helophoroides, EF- $1 \alpha$ was chosen as endogenous control in different development stages and tissues; $\alpha$-tubulin can be used as reference gene in adult $D$. helophoroides for different survival times. For each target gene and reference gene, specific primers were designed with Primer Premier 5.0 software (PREMIER Biosoft International, Palo Alto, CA, USA) (Table 1). Each pair of primers was validated by calculating standard curves with a $5 \times$ serial dilution of $D$. helophoroides $\mathrm{CDNA}$ as a template.

The expression of $D$. helophoroides mth-like transcripts was assayed by RT-qPCR using a Bio-rad IQ5 Thermol System with SYBR Green Mix (CWBIO, Beijing, China). Cycling conditions were as follows: $95^{\circ} \mathrm{C}$ for $3 \mathrm{~min}, 50 \mathrm{cycles}$ of $95^{\circ} \mathrm{C}$ for $30 \mathrm{~s}, 58^{\circ} \mathrm{C}$ for $30 \mathrm{~s}, 72{ }^{\circ} \mathrm{C}$ for $30 \mathrm{~s}$, followed by melting temperature analysis: $65-95{ }^{\circ} \mathrm{C}$ held for $10 \mathrm{~s}$ for each degree. The relative expression was determined using the $2^{-\Delta \Delta C t}$ method [34,35]. qPCR was repeated for a total of three biological replicates with three technical replicates each, which included a no template control and a no reverse-transcriptase control.

\subsection{Statistical Analysis}

All data were presented as mean \pm SD (standard deviation). Significant differences between each group were analyzed by using Tukey's test [36]; $p<0.05$ was considered statistically significant. One-way ANOVA was used for multiple comparisons using SPSS 20.0 (IBM SPSS Statistics, Chicago, IL, USA). 


\section{Results}

\subsection{Identification and Cloning of Putative Mth-Like Genes}

To facilitate the identification of mth-like transcripts in D. helophoroides, we searched the $D$. helophoroides transcriptome database. Three $D$. helophoroides unigenes homologous to insect $m$ th-like genes were identified in an assembly. To confirm the validity of the assembled transcripts, each putative mth-like was cloned and sequenced. Cloning and sequencing of full length cDNA of these mth-like sequences were accomplished using $5^{\prime}$ - and $3^{\prime}$-rapid amplification of cDNA ends (RACE). Gene-specific primers designed for each $m$ th-like sequence are shown in Table 1.

Three cDNA clones of $m$ th-like genes with full-length sequences were obtained. Determination of their putative amino acid sequences with Blastx tools showed that all three are members of the Mth-like (Mthl) family. The three nucleotide sequences were named as mth-like1, mth-like2, and mth-like5, and the corresponding proteins were, therefore, designated as Mth-like1, Mth-like2, and Mth-like5, due to their homology with Drosophila and T. castaneum proteins. Sequences were deposited in GenBank with accession numbers KM588897, KU363815, and KU363816. The full lengths of mth-like1, mth-like2, and mth-like5 were $2600 \mathrm{bp}, 1875 \mathrm{bp}$, and $2312 \mathrm{bp}$, which contained open reading frames (ORF) of $1701 \mathrm{bp}, 1353 \mathrm{bp}$ and, $1428 \mathrm{bp}$ and encoded proteins with 566, 450, and 475 amino acid residues, respectively. The deduced molecular weights were $63.8 \mathrm{kDa}, 51.8 \mathrm{kDa}$, and $54.4 \mathrm{kDa}$ and the isoelectric points (PI) were 8.67, 8.06, and 6.01, respectively (Table 2). All of these putative Mth-likes have conserved seven transmembrane domains, although the moderate degree of sequence similarity of Mth-like1, Mth-like2, and Mth-like5 compared with the Mth (NP-523871.1) in D. melanogaster are only $24 \%, 29 \%$, and $25 \%$, respectively (Figure 1 ).

Table 2. Basic sequence information of mth-like genes in Dastarcus helophoroides.

\begin{tabular}{ccccccc}
\hline $\begin{array}{c}\text { Gene } \\
\text { Name }\end{array}$ & Length (bp) & ORF (bp) & $\begin{array}{c}\text { Protein } \\
\text { Length (aa) }\end{array}$ & $\begin{array}{c}\text { Isoelectric } \\
\text { Point }\end{array}$ & $\begin{array}{c}\text { Molecular } \\
\text { Weight (kDa) }\end{array}$ & $\begin{array}{c}\text { Accession } \\
\text { Number }\end{array}$ \\
\hline mth-like1 & 2600 & 1701 & 566 & 8.67 & 63.8 & KM588897 \\
mth-like2 & 1875 & 1353 & 450 & 8.06 & 51.8 & KU363815 \\
mth-like5 & 2312 & 1428 & 475 & 6.01 & 54.4 & KU363816 \\
\hline
\end{tabular}


Drosophila melanogaster Mth Drosophila melanogaster Mth-like Drosophila melanogaster Mth-like Dastarcus helophoroides Mth-like Dastarcus helophoroides Mth-like

Drosophila melanogaster Mth Drosophila melanogaster Mth-like Drosophila melanogaster Mth-like 2 Drosophila melanogaster Mth-like Dastarcus helophoroides Mth-like Dastarcus helophoroldes Hith-like Dastarcus helophoroides Mth-like

Drosophila melanogaster Mth Drosophila melanogaster Mth-like Drosophila melanogaster Mth-like 2 Drosophila melanogaster Mth-like 5
Dastarcus helophoroides Mth-like 1 Dastarcus helophoroides Mth-like 2 Dastarcus helophoroldes Hth-like

Drosophila melanogaster Mth Drosophila melanogaster Mth-like Drosophila melanogaster Mth-like Dastarcus helophoroides Mth-like Dagcarcus helophoroides uth-1

Drosophila melanogaster Mth

Drosophila melanogaster Mth Drosophila melanogaster Mth-like 2 Drosophila melanogaster Mtholikester Mth-like Dastarcus helophoroides Mth-like Dastarcus helophoroides Hith-like Dastarcus helophoroides Mth-like

Drosophila melanogaster Mth Drosophila melanogaster Mth-like 1 Drosophila melanogaster Mth-like 2 Drosophila melanogaster Mth-like 5 Dastarcus helophoroides Mth-like 1 Dastarcus helophoroides Mth-like Dastarcus helophoroides Mth-like

Drosophila melanogaster Mth Drosophila melanogaster Mth-like 1 Drosophila melanogaster Mth-like Dastarcus helophoroides Mth-like Dastarcus helophoroides Mch-like Dastarcus helophoroides Mth-like

Drosophila melanogaster Mth Drosophila melanogaster Mth-like 1 Drosophila melanogaster Mth-like 2 Drosophila melanogaster Mth-like 5 Dastarcus helophoroides Mth-like 1 Dastarcus helophoroides Mth-like Drosophila melanogaster Mth-like Dastarcus helophoroldes Mth-like Drosophila melanogaster Mth-like Drosophila melanogaster Mth-like 5

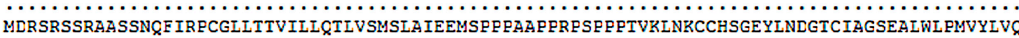

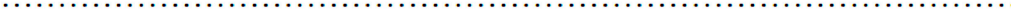
n.

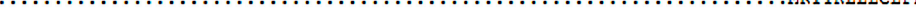
(n.

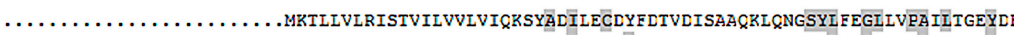

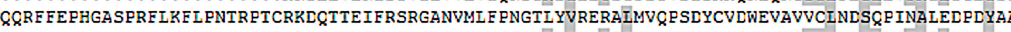

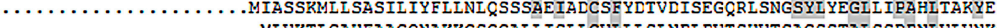
VVSGKNVII

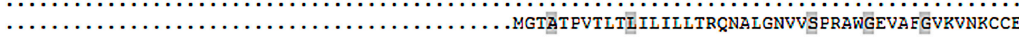

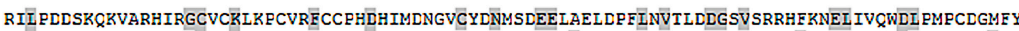

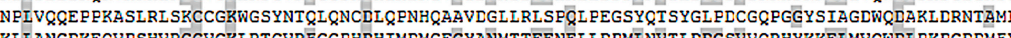
RIIANGDKECVPSHVRGCVCRIRTCVRFCCPHDHIMDMGECYANMTTEENE I I PPMINVTLDEGSVVQRHYKREIMVCWDL PRPCDDMF

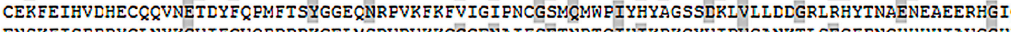

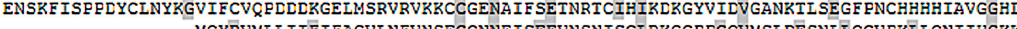
ENCINVGDSCTSSRLNETKWMPIFTDERGKSNIAVNYTFVTGL PDCGSRC IWPIYHYVGTDKITLL PNGVIRHYFDLHLIAAFEDEDE TM1

IDNREECLKYTL FENGTFFRHFDRVTIRKREYCLCQHLTFALGNATSIRIAPHNCL IVPSITGCTVVMISSLICMVLTIAVYLFVKKICN IDNREECLRYTLYCLTTFRHFDRVTLRRREYCICHITFALGNATSIRIAPHNCL IDNRDIMDEYTL FENGRLLRHYEQVYIDKSEYCLCHRTFGEGNNNSIRII I HNCL II PSRTGCTVVMITSIICIVLTIAVYICVRKIMN KLSKLFENGSIWISHYDTLVSMGNFCIEYSIENAGSPMVMVCSDTLRIEKP. TTDIRFVLYPIGIAFSAVELAATLAAGYLL PASH CENKS. AVLVSSSDTYKILELGCLYWRNSIYSYEDFCIENVDNCKIVVACVIETTEEDIANCIGMIIST PFIIATI IAYALL LDRNI SVMETLGCVRCYHDYHPSCYCMDKRIEDFT ....... SEFAMVCGPEHPTDWTATEYIMRNVVSPITHGLTILCILIIAIIYFIMPTIRD TM2 TM3

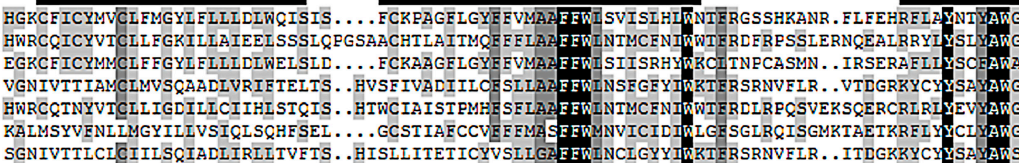

TM4

TM5

MAVVLTGITVLALNIVENC

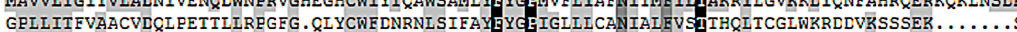
MELALTGVTYLADNVVNNEEWQPRVGLEGHCWIYTRSWSAMVY YYGEUVIIII FNITMEVITARHIIDSKRTIRRIARNEGR IQKLINSDK

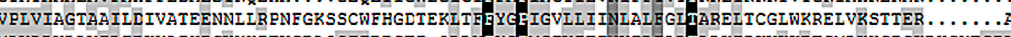
MAVLIVLAVIL IDKYA PHDSWYYHPEVGRACCFIRDGT . QLIY YYGEMGIVIT INVII HALTALKIRKVKKDTSMLKHSDSKRHSYESDK

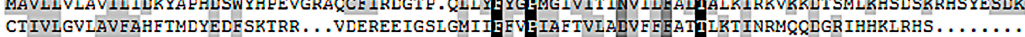
TM6 TM7

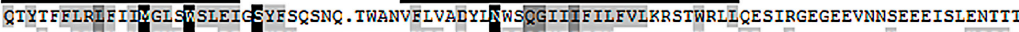
C. ANYT

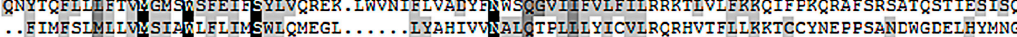

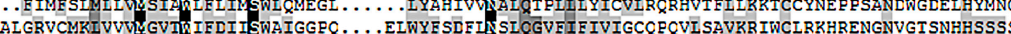

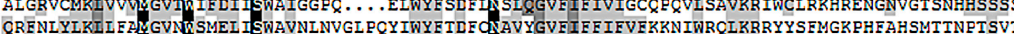

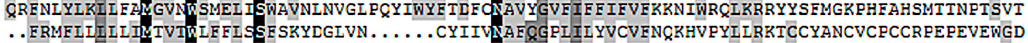

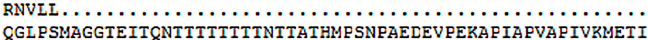
TRRHFNMT.

NLY. . .

CGLISIGLTMTNSVSNNTKSMPLETSC.

SYSTTVSNHDNANTDTSELTTRIS. . . SDYEEDIAGSIEPL YHDYDKGLYCIDKATSSTGEENVI FANICIARKEIRWSDSNFIIRKILNPIFHGISIVIILVIA IIYFIL TTI

65

180
67

66

103
0

45

155

270
157

156

193

135

245

360
247

246

282
161

219

330

450
331

332

370
247

305

420

532
421

411

453
336

384

509

618
510

493

539
426

426
466

514
675
518
496
566
450
475

Figure 1. Sequence alignment of Mth/Mth-likes. Sequences (cloned or annotated) are D. helophoroides Mth-likes (Mth-like1, Mth-like2, and Mth-like5), D. melanogaster Mth (NP-523871.1) and D. melanogaster Mth-likes (Mth-like1 NP-573140.1, Mth-like2 NP-788462.2, and Mth-like5 NP-650126.2). TM1-TM7 indicate the transmembrane domains. Identical or similar residues are highlighted black or grey, respectively, in the alignment. ORF: open reading frames.

\subsection{Phylogenetic Analysis of Mth-Like Genes}

To assess the relationships amongst the three D. helophoroides Mth-like sequences and those identified from other insects, phylogenetic analysis was carried out using the neighbor-joining (NJ) method implemented in MEGA 5 with default settings and bootstrap support based on 1000 iterations (Figure 2). The results showed that the total $18 \mathrm{Mth} / \mathrm{Mth}$-like proteins were divided into four clusters: Mth-like1, Mth-like2, Mth-like5, and Mth. Specifically, D. helophoroides Mth-likes (Mth-like1, Mth-like2, and Mth-like5) were more closely related to T. castaneum Mth-likes (Mth-like1, Mth-like2, and Mth-like5), showing $64 \%, 53 \%$, and $66 \%$ identity, respectively, with a bootstrap value of $100 \%$. 


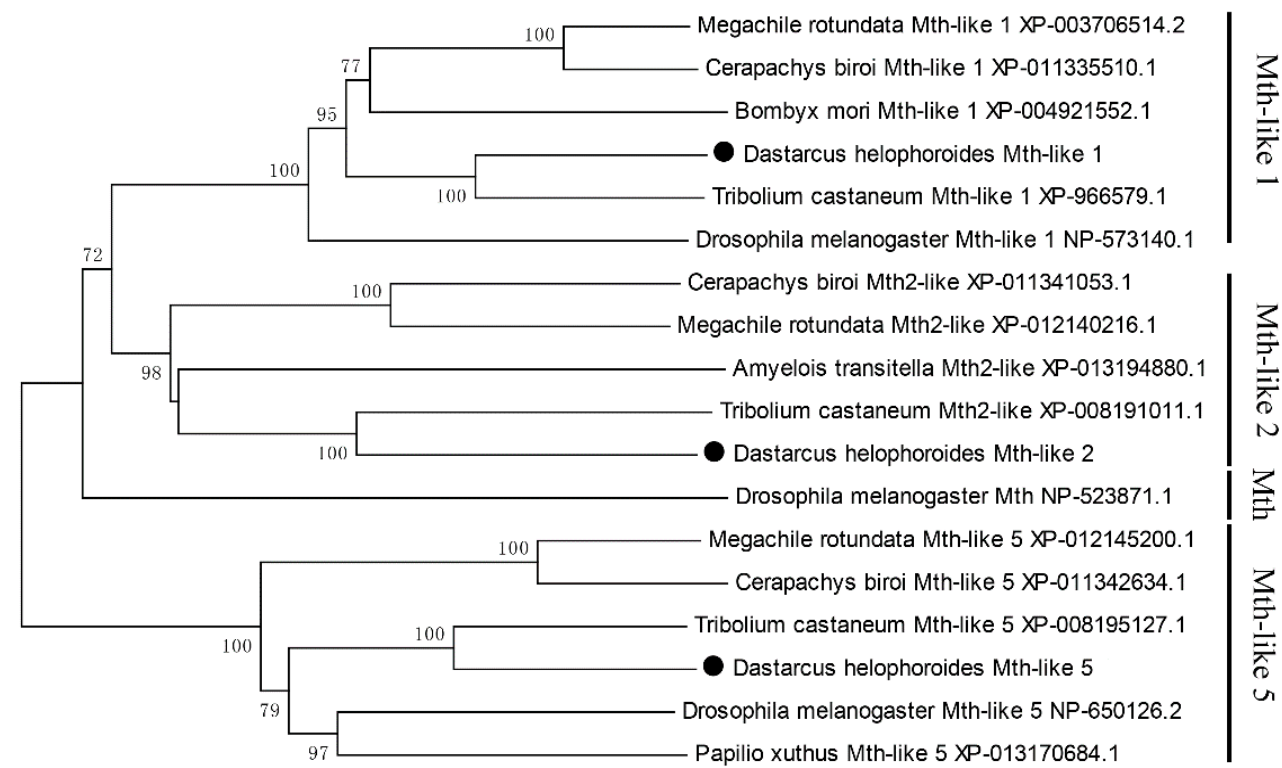

Figure 2. Molecular phylogenetic analysis by the neighbor-joining (NJ) method. Bootstrap values with 1000 trials re-indicated on the branches. GeneBank accession numbers of amino acid sequences used to generate the tree are shown in the Figure. D. helophoroides sequences are marked by a black circle.

\subsection{Developmental Expression Profiles}

To better understand the physiological roles of $D$. helophoroides mth-like genes in development, their expression profiles were examined by RT-qPCR at different life stages with EF- $1 \alpha$ as a reference gene. In general, $m$ th-likes are expressed throughout all the developmental stages examined, but they exhibited different expression patterns (Figure 3). The mRNA levels of $m$ th-like1 displayed a relatively high expression level at the late larval stage (fourth, fifth, and sixth larval stages) and mth-like 2 were high at the sixth larval and adult stages, while the mRNA levels of $m$ th-like 5 were highly expressed at the pupal and adult stages. The mRNA levels of all the three mth-like genes of female adults were significantly higher than that of males. These results showed that the three $m$ th-like genes were expressed differently during development indicating they might play different roles in the development of $D$. helophoroides.
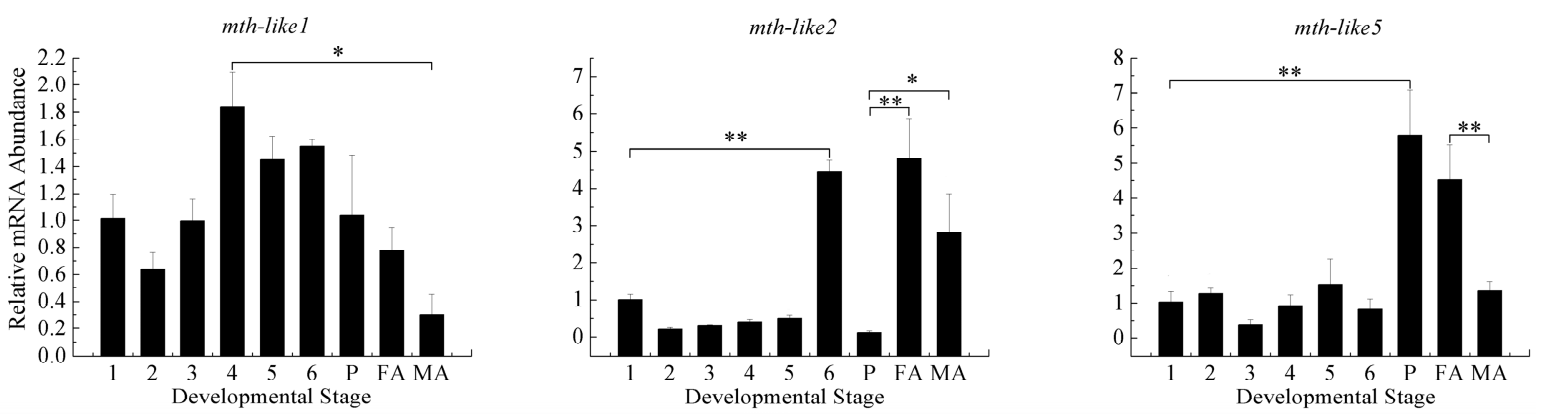

Figure 3. Developmental expression levels of $m$ th-like genes. The relative expression indicates the level of each mth-like transcript normalized to the internal standard of EF- $1 \alpha$, compared to expression levels of the first instar. Data are shown as the mean $\pm \mathrm{SD}$. Asterisks indicate differences that are statistically significant $\left({ }^{*}, p<0.05 ;{ }^{* *}, p<0.01\right) .1-6,1$ st-6th instar larvae; P, pupae; FA, female adults; MA, male adults. 


\subsection{Tissue Expression Profiles}

To explore tissue distribution, transcript abundance in the head, thorax, male and female reproductive systems, midgut, hindgut, fat body, and residual body were determined by RT-qPCR with EF- $1 \alpha$ as a reference gene. In general, although mth-like genes could be detectable in all of the examined tissues, they displayed different expression patterns and appeared to be specific (Figure 4). The expression levels of mth-like1 in the male and female reproductive system (15.80- and 16.86-fold relative to the head, respectively) were significantly higher than those in any other tissues. The expression levels of mth-like1 in the thorax and fat body (9.58- and 10.79-fold relative to the head, respectively) were significantly higher compared to those of the head, midgut, hindgut, and residual body, but significantly lower than in the male and female reproductive systems. The expression level of $m t h$-like 2 was the highest in the female reproductive system (56.78-fold relative to the head). Similarly, $m$ th-like 5 showed a significantly higher level in the female reproductive system (35.97-fold relative to the head), whilst the expression level in the residual body was significantly higher than in any other tissues (head, thorax, male reproductive systems, midgut, hindgut, and fat body), but lower than in the female reproductive system. These results demonstrated that these three $m$ th-like genes were differently expressed in the tissues and displayed high expression levels in the female reproductive system.
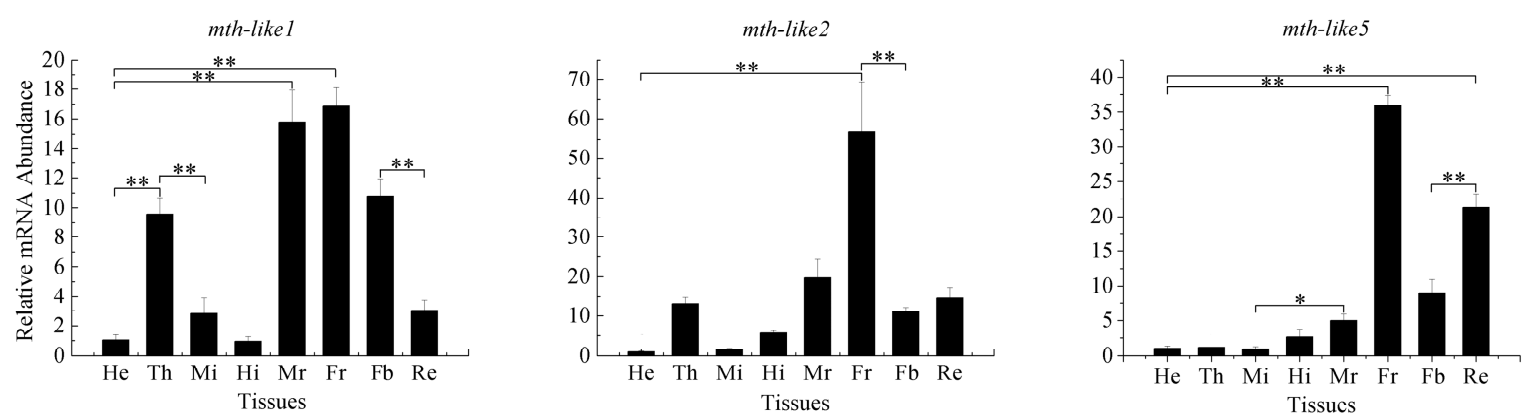

Figure 4. Tissue expression profiles of $m$ th-like genes. The relative expression indicates the level of each mth-like transcript normalized to the internal standard of EF-1 $\alpha$, compared to the expression level of head. Data are shown as the mean \pm SD. Asterisks indicate differences that are statistically significant $\left({ }^{*}, p<0.05 ;{ }^{* *}, p<0.01\right)$. He, head; Th, thorax; Mi, midgut; Hi, hindgut; $\mathrm{Mr}$, male reproductive system; $\mathrm{Fr}$, Female reproductive system; $\mathrm{Fb}$, fat body; $\mathrm{Rb}$, residual body.

\subsection{Expression Profiles during Aging Adults}

To explore the possible roles of the $m$ th-like genes in aging, expression levels of these three $m$ th-like genes in different age groups of adults were determined by RT-qPCR with $\alpha$-tubulin as a reference gene. As shown in Figure 5, the expression level of $m$ th-like1 increased significantly in the older groups (aged 20, 26, and 30 months), increased 2.74-fold for 20 months, 3.21-fold for 26 months, and 2.49-fold for 30 months, relative to the two-month group. The expression level of $m$ th-like 2 decreased from the two-month group, reaching the lowest point (15\% of the two-month group) in the 18-month group, and then increased in the older groups (aged 20,26, and 30 months), reaching a relatively high level (1.12-fold relative to the two-month group) in the 30-month group. The expression of $m$ th-like 5 remained relatively steady in the young groups (aged 2, 4, 10,12, and 18 months) and then significantly increased in the older groups (aged 20, 26, and 30 months), reaching the highest level (8.78-fold relative to the two-month group) in the 30-month group. Altogether, the expression levels of mth-like1 and $m$ th-like 5 were significantly increased in older groups (aged 20, 26, and 30 months) and mth-like5 displayed a more remarkable increase (4.28- to 8.78-fold) than mth-like1 (2.49- to 3.21-fold). Meanwhile, the expression level of $m$ th-like 2 began changing from the youngest adults (two-months) and recovered in the 30-month group. 

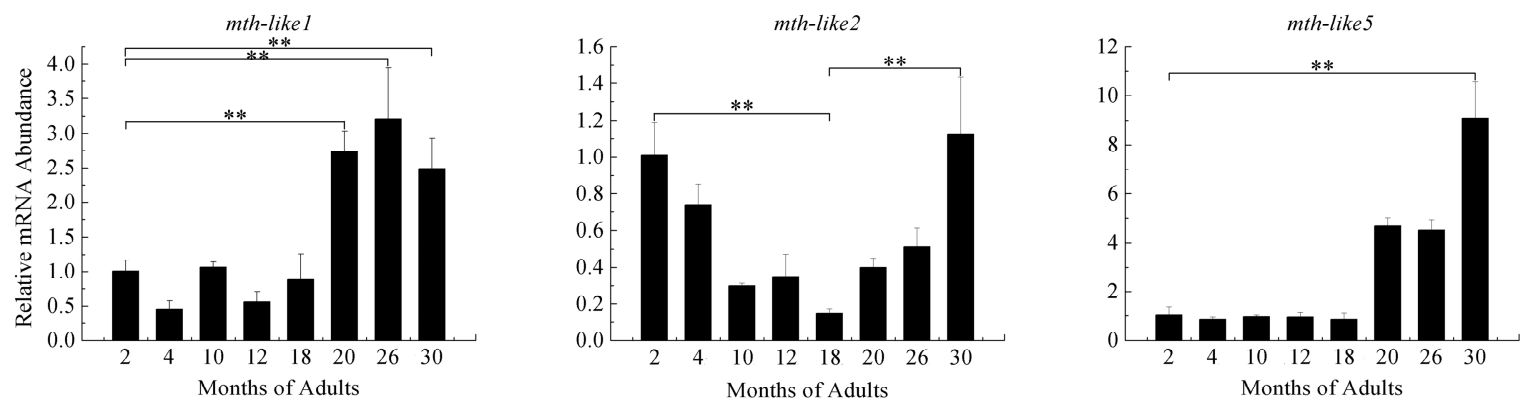

Figure 5. Expression of mth-like genes in different groups of adults (aged 2, 4, 10, 12, 18, 20, 26, and 30 months). The relative expression indicates the level of each $m$ th-like transcript normalized to the internal standard of $\alpha$-tubulin, compared to expression level of the first group (two-months). Data are shown as the mean \pm SD. Asterisks indicate differences that are statistically significant $\left({ }^{*}, p<0.05\right)$.

\subsection{Expression Profiles under the Oxidative Stress}

To determine whether $D$. helophoroides mth-like genes respond to oxidative stress, newly-emerged adults were exposed to paraquat and an abundance of these three $m$ th-like transcripts were determined over time after exposure by RT-qPCR with $\alpha$-tubulin as a reference gene. As shown in Figure 6, the expression levels of mth-like 1 in both male and female adults significantly down-regulated at the late stage ( $4-24 \mathrm{~h}$ for females, $12-24 \mathrm{~h}$ for males) of exposure, decreasing to $36.59 \%-58.62 \%$ of 0-h adults for females, and $22.36 \%-36.88 \%$ of 0 -h adults for males. Mth-like 1 expression levels in females were significantly higher when compared with males. On the whole, the expression levels of mth-like 2 in females were higher than that in males but this was not very significant. In female adults, the expression level of $m$ th-like 2 in female adults increased at the early stage of exposure, reaching the highest level (2.11-fold relative to the 0 -h group) at five hours before decreasing. While the expression in male adults displayed a relatively stable and high level (2.07-2.23-fold relative to the 0-h group) at the middle stage (3-6 h) of exposure, the expression level fluctuated and was higher than in males, but not very significantly. The expression of $m$ th-like 5 of females fluctuates in the early stage $(0-5 \mathrm{~h})$ of exposure and then was slightly down-regulated at the late stage $(6-24 \mathrm{~h})$. Furthermore, there is no significant variation in the expression level for males between all of the groups, but a higher expression level was found in females compared to males.
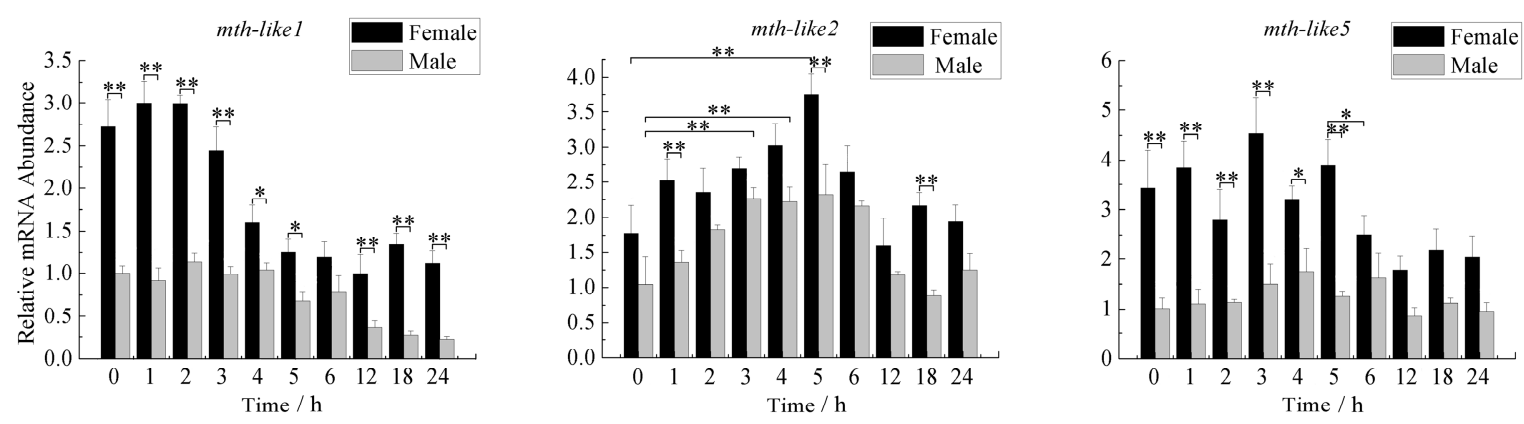

Figure 6. Expression profiles under oxidative stress. The relative expression indicates the level of each mth-like transcript normalized to the internal standard of $\alpha$-tubulin, compared to expression levels of the first group $(0 \mathrm{~h}) .0-24 \mathrm{~h}$ means different time under the oxidative stress. Data are shown as the mean $\pm \mathrm{SD}$. Asterisks indicate differences that are statistically significant $\left({ }^{*}, p<0.05 ;{ }^{*}, p<0.01\right)$.

\subsection{Expression Profiles under the Stress of High Temperature}

To determine whether $D$. helophoroides mth-like genes respond to high temperature stress, newly-emerged adults were exposed to a high temperature of $45^{\circ} \mathrm{C}$, and then the abundance of 
mth-like genes were detected both in males and females over time after eclosion with $\alpha$-tubulin as a reference gene. As shown in Figure 7, there is no obvious fluctuation in the expression level of the three $m$ th-like genes in either female or male adults with the exposure time increasing, while these three genes exhibited a relatively higher expression level in females than males. For $m$ th-like 1 in females over time, the expression of the gene was slightly increased at middle stages (1.5-2.0 h) compared to that at $1.0 \mathrm{~h}$. Simultaneously, it exhibited a slight decrease in males at the late exposure stage (2.0-12.5 h). The expression of mth-like 2 was significantly up-regulated in female adults (increased more than three-fold compared with the adults at $0-\mathrm{h})$, which was found at the middle stage $(1.5-2.0 \mathrm{~h})$ of exposure. Then, the expression decreased slowly as time went on at the late stage $(3.0-12.0 \mathrm{~h})$ of exposure, reaching the lowest level (2.06-fold relative to the 0 -h group) at $12.0 \mathrm{~h}$. Meanwhile, the expression of males increased at the early stage $(0-2.0 \mathrm{~h})$ and then decreased at the late stage $(2.0-12.0 \mathrm{~h}$ ) and peaked at $2.0 \mathrm{~h}$ (2.93-fold relative to the 0 -h group). Intriguingly, neither females nor males showed a significant variation in the expression level of $m$ th-like 5 among any of the groups.
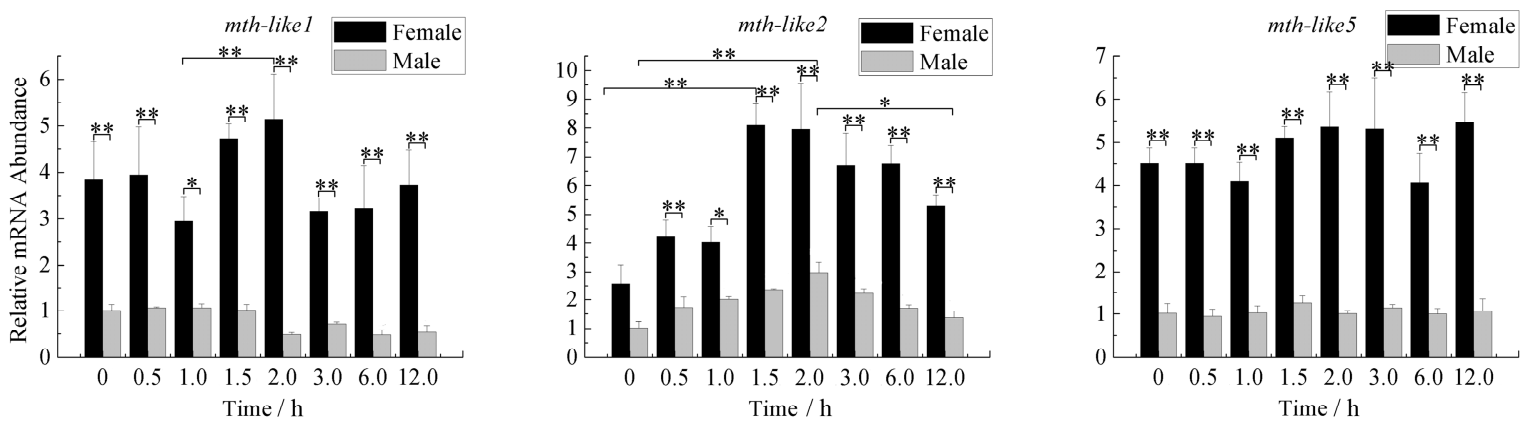

Figure 7. Expression profiles under the stress of high temperature. The relative expression indicates the level of each mth-like transcript normalized to the internal standard of $\alpha$-tubulin, compared to the expression level of the first group $(0 \mathrm{~h}) .0-12 \mathrm{~h}$ means different time of adults exposed at a high temperature of $45{ }^{\circ} \mathrm{C}$. Data are shown as the mean \pm SD. Asterisks indicate differences that are statistically significant $\left({ }^{*}, p<0.05 ;{ }^{* *}, p<0.01\right)$.

\subsection{Expression Profiles under the Stress of Starvation}

To determine whether $D$. helophoroides mth-like genes expression changed in response to food deprivation, newly-emerged adults (male and female) were cultured under the condition of no food and abundance of these three mth-like genes transcripts was determined by RT-qPCR over time with $\alpha$-tubulin as a reference gene. As shown in Figure 8, the expression level of mth-like 1 in both females and males showed no obvious regular fluctuation as time went on after exposure. However, the expression in females was significantly higher than in males. The expression of $m$ th-like 2 of females increased at the early stage (0-10 days), reaching the highest level (2.05-fold relative to the 0 -day group) 10 days after exposure, and then decreased to the normal level at 14 days (1.01-fold relative to 0 day) after exposure. In addition, the expression of $m$ th-like 2 in males shared a similar trend with that of females, but it peaked (1.91-fold relative to the 0-day group) six days after exposure and then returned to normal (1.12-fold relative to the 0 -day group) 14 days after exposure. The expression level in females was significantly higher than in males after exposure. The expression of $m$ th-like 5 in both females and males increased from the early stage ( $0-4$ days) to the highest level at (1.30-fold relative to the 0-day group for females, and 2.55-fold relative to the 0-day group for males, respectively) four days after exposure, and then it decreased with time to the bottom at 14 days, which showed a $30.37 \%$ and $41.35 \%$ reduction compared with the original levels (0-day adults) for female and male adults, respectively. The expression in females was higher than that of males at early and late stages, but not significantly at the early stage (0-10 days) after exposure. 

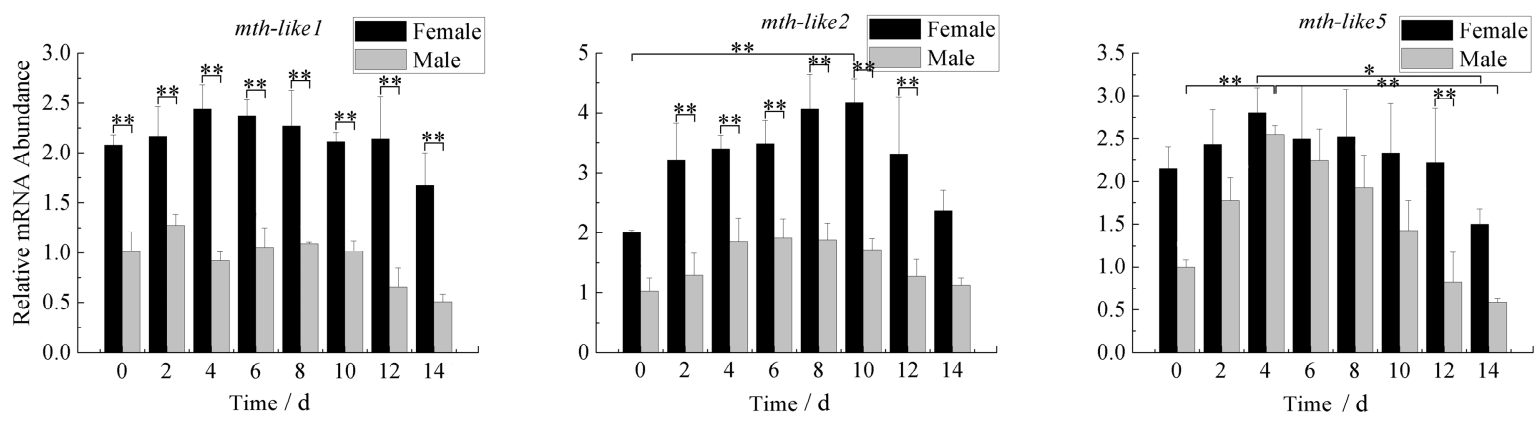

Figure 8. Expression profiles under the stress of starvation. The relative expression indicates the level of each $m$ th-like transcript normalized to the internal standard of $\alpha$-tubulin, compared to the expression level of the first group (0 day). 0-14 days means the starving time of adults. Data are shown as the mean \pm SD. Asterisks indicate differences that are statistically significant $\left({ }^{*}, p<0.05 ;{ }^{*}, p<0.01\right)$.

\section{Discussion}

In this study we identified and characterized three mth-like genes from $D$. melanogaster for the first time. The three genes were designated as mth-like1, mth-like2, and mth-like5, and the corresponding proteins were designated as Mth-like1, Mth-like2, and Mth-like5, respectively. Previous studies have demonstrated that $D$. melanogaster Mth-likes (homologous receptors of Mth) show high similarity to Mth and contain similar seven transmembrane $(7 \mathrm{tm})$ domains [3,37]. In our study, these three putative Mth-likes consisted of seven conserved transmembrane domains, which is consistent with the finding of a recent study in T. castaneum [38], although they only shared a moderate degree of sequence identity (24\%, 29\%, and 25\% for Mth-like1, Mth-like2, and Mth-like5, respectively) with the Mth (NP-523871.1) of D. melanogaster.

Phylogenetic analysis suggested that D. helophoroides Mth-likes (Mth-like1, Mth-like2, and Mth-like5) were clustered into Mth-like1, Mth-like2, and Mth-like5 groups, respectively, and are very close to T. castaneum Mth-likes (Mth-like1, Mth-like2, and Mth-like5) in the tree, showing 64\%, $53 \%$, and $66 \%$ identity, respectively. A recent study on the expression pattern and phylogenetic relationship of some $m$ th-likes/mth in D. melanogaster and T. castaneum suggested subfunctionalization and acquisition of novel functionalities [3], so the functional divergence most likely occurred between $D$. melanogaster $m$ th and $D$. helophoroides mth-like genes, while $D$. helophoroides Mth-likes were likely to have similar functions with T. castaneum Mth-likes. Recent research has revealed that the $M t h / M t h l$ gene family is ancient, and Mthl1, Mthl5, Mthl14, and that Mthl15 are the oldest Mth/Mthl gene family paralogs in Drosophila [39]. However, it is hard to judge which one is the oldest through phylogenetic analysis in this study, so more detailed analysis and further research is needed.

A diversity in expression patterns for these three $m t h$-like genes from $D$. helophoroides was also found in this study. The expression of mth-like1 displayed a relative high level at the late larval stage (fourth, fifth, and sixth larval stages), mth-like2 at the sixth larval and adult stages, and mth-like5 at the pupal and adult stages (Figure 3). Previous studies in D. melanogaster have shown that $m$ th was expressed in both embryos and third-instar central nervous system (CNS) or discs, mth-like1 and mth-like 5 were expressed in the embryo, while mth-like 2 was not detected in whole embryos or third-instar brain and imaginal discs using in situ hybridization techniques [3]. A recent study suggested that the mRNA levels of mth-like1 of T. castaneum were highly expressed at the late egg, pupa, and early adult stage, and mth-like 2 and $m$ th-like 5 were highly expressed at the early embryonic and late pupal stages [38]. Similarly to T. castaneum mth-like genes, mth-like genes of D. helophoroides were expressed throughout all of the larval, pupal, and adult stages, but to some extent they display different expression patterns, indicating that $m$ th-like genes ( $m$ th-like2 and $m$ th-like5) of $D$. helophoroides were likely to have similar functions involved in larval and pupal development and the process of 
eclosion to T. castaneum mth-like genes, while functional divergence most likely occurred between T. castaneum and D. helophoroides mth-like genes (mth-like1).

In addition to development, mth-like1 and mth-like2 are also involved in reproduction in T. castaneum. In the same recent study, when the expression of $m$ th-like genes ( $m$ th-like1 and $m$ th-like2) was inhibited, one pair of beetles laid less eggs per day, but mth-like 5 was not involved in fertility [38]. Previous studies have shown that the fertility of $m$ th-mutated flies is decreased between $25^{\circ} \mathrm{C}$ and $29^{\circ} \mathrm{C}$ [11]. In our study, the mRNA levels of $m$ th-like1, $m$ th-like2, and $m$ th-like 5 were all highly expressed in the female reproductive system (Figure 4). Tissue expression profiles of $m$ th-like genes demonstrate that $m$ th-like genes are likely to be involved in reproduction in D. helophoroides and high expression levels of mth-like genes are likely to be crucial for normal fertility. Meanwhile, further research on mth-like genes in D. helophoroides is needed to explore whether they are functionally consistent in reproduction with T. castaneum mth-like genes.

Existing studies have demonstrated that both mutation and suppression of $m$ th can enhance the longevity of $D$. melanogaster $[1,39]$. Conversely, the $T$. castaneum groups with suppression of mth-like1, mth-like2, and mth-like5 had a significantly shorter lifespan, which applied to both sexes [38], indicating that both $m$ th and $m$ th-like genes are involved in insect aging and are functionally divergent. However, both $m$ th mutation in $D$. melanogaster and $m$ th-like gene suppression in $T$. castaneum have been reported to result in lethality for pre-adults [1,38], which demonstrates that at least some activity of the $m t h$ and $m$ th-like genes are essential for survival. In our study, the expression levels of both $m$ th-like1 and mth-like 5 increased significantly in the older groups (aged 20,26, and 30 months), while mth-like2 firstly decreased, and then increased, finally recovering to the normal level (Figure 5). This may demonstrate that $m$ th-like genes are involved in aging and are likely to have positive effects on lifespan of $D$. helophoroides. To some extent, the normal or overexpression of $m$ th-like genes is likely to be the reason why $D$. helophoroides adults have a long lifespan.

Reduced signaling via Methuselah (Mth), a G-protein coupled receptor (GPCR) required for neurosecretion, has previously been reported to enhance stress resistance in flies [1,40]. A previous study on mth-like genes of $T$. castaneum showed that suppression of $m$ th-like genes decreased resistance to the stress of oxidation (mth-like5), high temperature (mth-like1 and mth-like2), and starvation (mth-like1, mth-like2, mth-like3, mth-like4, and mth-like5) [38]. In our study, the mRNA levels of mth-like1, $m$ th-like2, and $m$ th-like5 were all responsive to the stress of paraquat (Figure 6), only $m$ th-like 2 was responsive to the stress of high temperature (Figure 7), and mth-like 2 and $m$ th-like 5 were responsive to starvation stress (Figure 8). This demonstrates that both $m$ th and $m$ th-like genes are likely to be involved in stress resistance and functional divergence may have occurred among insect $m$ th and mth-like genes. A recent study on the expression pattern and phylogenetic relationship of some $m$ th $/ m$ th-like genes in D. melanogaster and T. castaneum suggested subfunctionalization and acquisition of novel functionalities [3].

Both reduced expression and overexpression of $m$ th targeted to the insulin producing cells (IPCs) of the fly brain were able to extend life and enhance oxidative stress resistance due to Mth's interaction with $\beta$-arrestin, which uncouples GPCRs from their G-proteins [1]. Enhanced longevity and stress resistance in Caenorhabditis elegans and flies are often the result of signals from several pathways integrated and coordinated by activation of the forkhead box O (FOXO) transcription factor [41-44]. The lifespan and oxidative stress effects of MTH signaling are FOXO-dependent and rely not only on the abundance of Mth itself, but also on the abundance of the $\beta$-arrestin scaffold protein, which interacts with Mth to inhibit its signaling in critical cellular targets [1]. In summary, this is consistent with a mechanism by which reduced MTH signaling increases longevity and enhances stress resistance by directly reducing systemic insulin-IGF (insulin-like growth factor) signaling (IIS), as it has previously been shown in worms and mice, as well as flies [45-49]. In the present research the regular changes of $m$ th-like genes mRNA levels in D. helophoroides adults of different age groups (Figure 5), under the stress of oxidation (Figure 6), high temperature (Figure 7), and starvation (Figure 8), are likely the result of Mth-likes' interaction with other factors. This demonstrates that $m$ th-like genes are likely to be 
involved in a complex mechanism by which changed Mth-likes' signaling affects longevity and the ability of stress resistance.

\section{Conclusions}

Three $m$ th-like genes were identified from $D$. helophoroides, which showed different transcriptional expression profiles at different developmental stages, in various tissues, and in different age groups of adults, as well as in response to the stress of oxidation, high temperature, and starvation. Our findings provide a theoretical basis for further research in molecular biology analysis of D. helophoroides, and establish valuable insights for further investigation into the functions of $m$ th-likes in insects in the future.

Acknowledgments: This work was funded by the National Natural Sciences Foundation of China (No. 31170608) and the research initiation funds for introduced talent of Shaanxi Xueqian Normal University (No. 2014DS026).

Author Contributions: Zhengqing Zhang and Huapeng Wang contributed equally to this study. Huapeng Wang, Zhengqing Zhang and Menglou Li designed the research. Zhengqing Zhang made best efforts in data collection and analysis and writing in the earlier version and revisions. Huapeng Wang was responsible for data collection, data analysis, and drafted the earlier versions of the manuscript. Chunfeng Hao was involved in the data analysis. Zhengqing Zhang, Wei Zhang and Miaomiao Yang revised the manuscript. Yong Chang was involved in sample collection. All authors have read and approved the final manuscript.

Conflicts of Interest: The authors declare no conflict of interest.

\section{References}

1. Lin, Y.J.; Seroude, L.; Benzer, S. Extended life-span and stress resistance in the Drosophila mutant methuselah. Science 1998, 282, 943-946. [CrossRef] [PubMed]

2. Fredriksson, R.; Lagerström, M.C.; Lundin, L.G.; Schiöth, H.B. The G-protein-coupled receptors in the human genome form five main families. Phylogenetic analysis, paralogon groups, and fingerprints. Mol. Pharmacol. 2003, 63, 1256-1272. [CrossRef] [PubMed]

3. Patel, M.V.; Hallal, D.A.; Jones, J.W.; Bronner, D.N.; Zein, R.; Caravas, J.; Husain, Z.; Friedrich, M.; Vanberkum, M.F.A. Dramatic expansion and developmental expression diversification of the methuselah gene family during recent Drosophila evolution. J. Exp. Zool. B Mol. Dev. Evol. 2012, 318, 368-387. [CrossRef] [PubMed]

4. Brody, T.; Cravchik, A. Drosophila melanogaster G protein-coupled receptors. J. Cell Biol. 2000, 150, F83-F88. [CrossRef] [PubMed]

5. West, A.P.; Llamas, L.L.; Snow, P.M.; Benzer, S.; Bjorkman, P.J. Crystal structure of the ectodomain of Methuselah, a Drosophila G protein-coupled receptor associated with extended lifespan. Proc. Natl. Acad. Sci. USA 2001, 98, 3744-3749. [CrossRef] [PubMed]

6. Harmar, A.J. Family-B G-protein-coupled receptors. Genome Biol. 2001, 2, 3013.1-3013.10. [CrossRef]

7. Aigaki, T.; Seong, K.H.; Matsuo, T. Longevity determination genes in Drosophila melanogaster. Mech. Ageing Dev. 2002, 123, 1531-1541. [CrossRef]

8. Grace, C.R.; Perrin, M.H.; Gulyas, J.; Digruccio, M.R.; Cantle, J.P.; Rivier, J.E.; Vale, W.W.; Riek, R. Structure of the N-terminal domain of a type B1 G protein-coupled receptor in complex with a peptide ligand. Proc. Natl. Acad. Sci. USA 2007, 104, 4858-4863. [CrossRef] [PubMed]

9. Parthier, C.; Kleinschmidt, M.; Neumann, P.; Rudolph, R.; Manhart, S.; Schlenzig, D.; Fanghanel, J.; Rahfeld, J.U.; Demuth, H.U.; Stubbs, M.T. Crystal structure of the incretin-bound extracellular domain of a G protein-coupled receptor. Proc. Natl. Acad. Sci. USA 2007, 4, 13942-13947. [CrossRef] [PubMed]

10. Sohal, R.S. Role of oxidative stress and protein oxidation in the aging process. Free Radic. Biol. Med. 2002, 33, 37-44. [CrossRef]

11. Mockett, R.J.; Sohal, R.S. Temperature-dependent trade-offs between longevity and fertility in the Drosophila mutant, methuselah. Exp. Gerontol. 2006, 41, 566-573. [CrossRef] [PubMed]

12. Song, W.; Ranjan, R.; Dawson, S.K.; Bronk, P.; Marin, L.; Seroude, L.; Lin, Y.J.; Nie, Z.; Atwood, H.L.; Benzer, S.; et al. Presynaptic regulation of neurotransmission in Drosophila by the G protein-coupled receptor methuselah. Neuron 2002, 36, 105-119. [CrossRef] 
13. Lee, K.P.; Simpson, S.J.; Clissold, F.J.; Brooks, R.; Ballard, J.W.O.; Taylorp, W.; Soran, N.; Raubenheimer, D. Lifespan and reproduction in Drosophila: New insights from nutritional geometry. Proc. Natl. Acad. Sci. USA 2007, 7, 2498-2503. [CrossRef] [PubMed]

14. Bjedov, I.; Toivonen, J.M.; Kerr, F.; Slack, C.; Jacobson, J.; Foley, A.; Partridge, L. Mechanisms of life span extension by Rapamycin in the fruit fly Drosophila melanogaster. Cell Metab. 2010, 11, 35-46. [CrossRef] [PubMed]

15. Wallenfang, M.R.; Nayak, R.; Nardo, S.D. Dynamics of the male germline stem cell population during aging of Drosophila melanogaster. Aging Cell 2006, 5, 297-304. [CrossRef] [PubMed]

16. Petrosyan, A.; Hsieh, I.H.; Saberi, K. Age-dependent stability of sensorimotor functions in the life-extended Drosophila mutant Methuselah. Behav. Genet. 2007, 37, 585-594. [CrossRef] [PubMed]

17. Hill, C.A.; Fox, A.N.; Pitts, R.J.; Kent, L.B.; Tan, P.L.; Chrystal, M.A.; Cravchik, A.; Collins, F.H.; Robertson, H.M.; Zwiebel, L.J. G protein-coupled receptors in Anopheles gambiae. Science 2002, 298, 176-178. [CrossRef] [PubMed]

18. Fan, Y.; Sun, P.; Wang, Y.; He, X.B.; Deng, X.Y.; Chen, X.P.; Zhang, G.Z.; Chen, X.; Zhou, N.M. The G protein-coupled receptors in the silkworm, Bombyx mori. Insect Biochem. Mol. Biol. 2010, 40, 581-591. [CrossRef] [PubMed]

19. Bai, H.; Zhu, F.; Shah, K.; Palli, S.R. Large-scale RNAi screen of G protein-coupled receptors involved in larval growth, molting and metamorphosis in the red flour beetle. BMC Genom. 2011. [CrossRef] [PubMed]

20. Li, C.; Chen, M.; Sang, M.; Liu, X.; Wu, W.; Li, B. Comparative genomic analysis and evolution of family-B $\mathrm{G}$ protein-coupled receptors from six model insect species. Gene 2013, 519, 1-12. [CrossRef] [PubMed]

21. Wei, J.R.; Yang, Z.Q.; Poland, T.M.; Du, J.W. Parasitism and olfactory responses of Dastarcus helophoroides (Coleoptera: Bothrideridae) to different Cerambycid hosts. Biocontrol 2009, 54, 733-742. [CrossRef]

22. Wang, X.M.; Ren, G.D.; Ma, F. Classification position of Dastarcus helophoroides and its applied prospects. Acta Agric. Bor-Occid. Sin. 1996, 5, 75-78.

23. Zhang, Y.N.; Yang, Z.Q. Studies on the natural enemies and biocontrol of Monochamus alternatus Hope (Coleoptera: Cerambycidae). Plant Prot. 2006, 32, 9-14.

24. Li, M.L.; Wang, P.X.; Ma, F.; Yang, Z.Q. Study on the Parasitic Efficiency of Dastarcus helophoroides on Anoplophora glabripennis. Available online: http://en.cnki.com.cn/Article_en/CJFDTOTAL-XBNY200706030. htm (accessed on 15 October 2016). (In Chinese)

25. Wang, H.D.; Li, F.F.; He, C.; Cui, J.; Song, W.; Li, M.L. Molecular cloning and sequence analysis of novel cytochrome P450 cDNA fragments from Dastarcus helophoroides. J. Insect Sci. 2014. [CrossRef] [PubMed]

26. Lei, Q.; Li, M.L.; Yang, Z.Q. A study on biological feature of Dastarcus longulus. J. Northwest Sci.-Tech. Univ. Agric. For. (Natl. Sci. Ed.) 2003, 31, 62-66. (In Chinese)

27. Zhang, W.; Song, W.; Zhang, Z.Q.; Wang, H.D.; Yang, M.M.; Guo, R.J.; Li, M.L. Transcriptome Analysis of Dastarcus helophoroides (Coleoptera: Bothrideridae) Using Illumina HiSeq Sequencing. PLoS ONE 2014, 9, e100673. [CrossRef] [PubMed]

28. BLAST, BlastX. Available online: https://blast.ncbi.nlm.nih.gov/Blast.cgi?PROGRAM=blastx\&PAGE_ TYPE=BlastSearch\&LINK_LOC=blasthome (accessed on 15 October 2016).

29. National Center of Biotechnology Information. Available online: https://www.ncbi.nlm.nih.gov/ (accessed on 15 October 2016).

30. ORFfinder. Available online: https://www.ncbi.nlm.nih.gov/orffinder/ (accessed on 15 October 2016).

31. Qiao, L.; Wu, J.X.; Qin, D.Z.; Lu, Z.C.; Pan, Z.L.; Chen, H.; Li, G.W. Gene Expression profiles of heat shock proteins 70 and 90 from Empoasca onukii (Hemiptera: Cicadellidae) in response to temperature stress. J. Insect Sci. 2015, 15, 49-49. [CrossRef] [PubMed]

32. ExPASy, Compute pI/Mw Tool. Available online: http://web.expasy.org/compute_pi/ (accessed on 15 October 2016).

33. Tamura, K.; Peterson, D.; Peterson, N.; Stecher, G.; Nei, M.; Kumar, S. MEGA5: Molecular evolutionary genetics analysis using maximum likelihood, evolutionary distance, and maximum parsimony methods. Mol. Biol. Evol. 2011, 28, 2731-2739. [CrossRef] [PubMed]

34. Livak, K.J.; Schmittgen, T.D. Analysis or relative gene expression data using real-time quantitative PCR and the 2- $\Delta \Delta C$ T method. Methods 2001, 25, 402-408. [CrossRef] [PubMed]

35. Pfaffl, M.W. A new mathematical model for relative quantification in real-time RT-PCR. Nucleic Acids Res. 2001. [CrossRef] 
36. Dai, L.; Wang, C.; Zhang, X.; Yu, J.; Zhang, R.; Chen, H. Two CYP4 genes of the Chinese white pine beetle, Dendroctonus armandi (Curculionidae: Scolytinae), and their transcript levels under different development stages and treatments. Insect Mol. Biol. 2014, 23, 598-610. [CrossRef] [PubMed]

37. Paaby, A.B.; Schmidt, P.S. Functional significance of allelic variation at methuselah, an aging gene in Drosophila. PLoS ONE 2008, 3, e1987. [CrossRef] [PubMed]

38. Li, C.J.; Zhang, Y.; Yun, X.P.; Wang, Y.Y.; Sang, M.; Liu, X.; Hu, X.X.; Li, B. Methuselah-like genes affect development, stress resistance, lifespan and reproduction in Tribolium castaneum. Insect Mol. Biol. 2014, 23, 587-597. [CrossRef] [PubMed]

39. Mendoza, A.; Jones, J.W.; Friedrich, M. Methuselah/Methuselah-like G protein-coupled receptors constitute an ancient metazoan gene family. Sci. Rep. 2016. [CrossRef] [PubMed]

40. Gimenez, L.E.; Ghildyal, P.; Fischer, K.E.; Hu, H.X.; Ja, W.W.; Eaton, B.A.; Wu, Y.M.; Austad, S.N.; Ranjan, R. Modulation of methuselah expression targeted to Drosophila insulin-producing cells extends life and enhances oxidative stress resistance. Aging Cell 2013, 12, 121-129. [CrossRef] [PubMed]

41. Hwangbo, D.S.; Gershman, B.; Tu, M.P.; Palmer, M.; Tatar, M. Drosophila dFOXO controls lifespan and regulates insulin signalling in brain and fat body. Nature 2004, 429, 562-566. [CrossRef] [PubMed]

42. Wang, M.C.; Bohmann, D.; Jasper, H. JNK extends life span and limits growth by antagonizing cellular and organism-wide responses to insulin signaling. Cell 2005, 121, 115-125. [CrossRef] [PubMed]

43. Broughton, S.; Partridge, L. Insulin/IGF-like signalling, the central nervous system and aging. Biochem. J. 2009, 418, 1-12. [CrossRef] [PubMed]

44. Hay, N. Interplay between FOXO, TOR, and Akt. Biochim. Biophys. Acta 2011, 1813, 1965-1970. [CrossRef] [PubMed]

45. Apfeld, J.; Kenyon, C. Regulation of lifespan by sensory perception in Caenorhabditis elegans. Nature 1999, 402, 804-809. [PubMed]

46. Clancy, D.J.; Gems, D.; Harshman, L.G.; Oldham, S.; Stocker, H.; Hafen, H.; Leevers, S.J.; Partridge, L. Extension of life-span by loss of CHICO, a Drosophila insulin receptor substrate protein. Science 2001, 292, 104-106. [CrossRef] [PubMed]

47. Tatar, M.; Kopelman, A.; Epstein, D.; Tu, M.P.; Yin, C.M.; Garofalo, R.S. A mutant Drosophila insulin receptor homolog that extends life-span and impairs neuroendocrine function. Science 2001, 292, 107-110. [CrossRef] [PubMed]

48. Ikeya, T.; Galic, M.; Belawat, P.; Nairz, K.; Hafen, E. Nutrient-dependent expression of insulin-like peptides from neuroendocrine cells in the CNS contributes to growth regulation in Drosophila. Curr. Biol. 2002, 12, 1293-1300. [CrossRef]

49. Bluher, M.; Kahn, B.B.; Kahn, C.R. Extended longevity in mice lacking the insulin receptor in adipose tissue. Science 2003, 299, 572-574. [CrossRef] [PubMed]

(C) 2016 by the authors; licensee MDPI, Basel, Switzerland. This article is an open access article distributed under the terms and conditions of the Creative Commons Attribution (CC-BY) license (http://creativecommons.org/licenses/by/4.0/). 